\title{
Micobiota produtora de fitase isolada de solo e serapilheira do Bioma Cerrado
}

\author{
Phytase producing mycobiota isolated from soil and litter of Cerrado Biome \\ Alexandre De Donato ${ }^{\mathrm{I}}$, Tatiana Faria Maia ${ }^{\mathrm{II}}$, Tiago de Conto ${ }^{\mathrm{III}}$, Marcos Gervasio \\ Pereira $^{\text {Iv }}$, Marcelo Elias Fragav
}

\begin{abstract}
Resumo
Grande parte dos solos agricultáveis brasileiros apresenta deficiência de $\mathrm{P}$, em função da maior parte deste elemento estar indisponível para a absorção pelas plantas. Neste contexto, a habilidade dos fungos filamentosos em solubilizar o P de várias fontes, sejam estas, insolúveis, orgânicas e/ou inorgânicas, tem sido considerada uma característica de destacada importância. O objetivo do presente estudo foi caracterizar a micobiota produtora de fitase no Bioma Cerrado, bem como, apresentar uma caracterização dos fatores abióticos do presente bioma e as possíveis correlações com a micobiota encontrada. As amostras foram coletadas de oito fragmentos florestais, em duas épocas, duas profundidades do solo $(0-5$ e $5-10 \mathrm{~cm})$ e na camada de serapilheira para a quantificação da micobiota e avaliação da produção de fitase. As coletas foram realizadas em duas épocas, sendo uma na época chuvosa e outra na época seca. Na coleta da época chuvosa, o número total de fungos produtores de fitase foi menor, mas o número de Aspergillus seção Nigri produtores de fitase foi maior. Na época seca, o número de fungos produtores de fitase pertencentes ao gênero Penicillium foi maior. $O$ fator época do ano alterou qualitativamente e quantitativamente a micobiota produtora de fitase. Concluiu-se que os solos do Bioma Cerrado são potencialmente exploráveis para a obtenção de fungos solubilizadores de $\mathrm{P}$, sendo a época seca do ano a mais propícia para este fim. Palavras-chave: Fungos; Penicillium; Trichoderma; Aspergillus
\end{abstract}

\begin{abstract}
The majority of Brazilian arable soils shows P deficiency, due to the fact that most of this element in the soil is unavailable for the plant absorption, for this and other reasons, the ability of filamentous fungi to solubilize P from various sources, insoluble, organic and inorganic, has been considered a desirable feature for several uses. The aim of this study was to characterize the production of phytase mycobiota in soil located in Cerrado in two seasons, under dry and rainy seasons, and also characterize abiotic factors in this biome in order to verify the existence of correlations between the quantitative and qualitative aspects of their mycobiotas in these conditions. The samples were collected from eight forest fragments twice, two soil depths $(0-5$ and $5-10 \mathrm{~cm})$ and litter layer to quantify mycobiota and the evaluation of phytase production. In the collection carried out in the rainy season, the total number of phytase producing fungi was lower, but the number of Aspergillus section Nigri producers of phytase was greater. In the dry season the number of phytase producing fungi belonging to the genus Penicillium was higher. The year season's factor alters quantitatively and qualitatively the phytase producer mycobiota. It was concluded that the soils are potentially exploitable in relation to obtaining phosphorus solubilizing fungi, and the dry season is more propitiate to the achievement of these fungi.
\end{abstract}

Keywords: Fungi; Aspergillus; Trichoderma; Penicillium

Engenheiro Agrônomo, MSc., Universidade Federal de Rural do Rio de Janeiro, BR 465, KM 07, CEP 23890-000, Seropédica (RJ), Brasil.

alexandre_ulianov@hotmail.com (ORCID: 0000-0002-6514-2654)

Bióloga, MSc., Universidade Federal de Rural do Rio de Janeiro, BR 465, KM 07, CEP 23890-000, Seropédica (RJ), Brasil.

tatiana.fariamaia@gmail.com (ORCID: 0000-0002-6902-1817)

III Engenheiro Florestal, MSc., Centro AgTech Coworking Piracicaba - SP, Rua Tiradentes, 435, CEP 13.400-760, Piracicaba (SP), Brasil. ti@forlidar.com.br (ORCID: 0000-0003-1827-829X)

IV Engenheiro Agrônomo, Dr., Professor Titular do Departamento de Solos, Universidade Federal de Rural do Rio de Janeiro, BR 465, KM 07, CEP 23890-000, Seropédica (RJ), Brasil. gervasio@ufrrj.br (ORCID: 0000-0002-1402-3612)

Biólogo, Dr., Professor Associado IV do Departamento de Microbiologia e Imunologia Veterinária, Universidade Federal de Rural do Rio de Janeiro, BR 465, KM 07, CEP 23890-000, Seropédica (RJ), Brasil. fraga@ufrrj.br (ORCID: 0000- 0002-6948-6032) 


\section{Introdução}

Os solos das regiões tropicais são, de maneira geral, naturalmente ácidos, devido ao alto grau de intemperização, que resulta em maior transformação dos minerais primários em minerais secundários, favorecendo o aumento dos teores de minerais como a caulinita e os óxidos, hidróxidos, oxi-hidróxidos de Fe e Al. Os óxidos de ferro, por serem coloides de carga variável, podem apresentar uma forte afinidade com $P$, quando grande parte da sua superfície encontrase carregada positivamente, o que faz com que o $\mathrm{P}$ se ligue covalentemente, diminuindo sua disponibilidade no solo (SOARES; ALLEONI, 2008). Segundo a International Fertilizes Industry Association (2006), são usados mais de 30 milhões de $\mathrm{Mg}$ /ano de fertilizantes fosfatados nos solos do mundo inteiro. Com base no consumo atual de fertilizantes, estima-se que a fonte de $\mathrm{P}$ esteja esgotada em aproximadamente 90 anos (GUSEWELL, 2004). O Cerrado tem como característica climática verões chuvosos e invernos secos, temperaturas médias entre $21,3^{\circ} \mathrm{C}$ e $27,2^{\circ} \mathrm{C}$ e precipitação de $1500 \mathrm{~mm}+/-500 \mathrm{~mm}$, e pode atingir, em algumas regiões e épocas do ano, UR abaixo de $10 \%$. A maioria dos solos presentes neste bioma é classificado como Latossolo, de relevo plano a leve ondulado, e distróficos. Considerando que o Cerrado é o segundo maior bioma presente no Brasil, ocupando mais de $20 \%$ do território nacional, presente em 10 Estados, e que $60 \%$ da expansão da fronteira agrícola nos últimos 40 anos se deu neste bioma, pode-se perceber o quanto o desenvolvimento de tecnologias ligadas à fertilidade do solo é importante para o desenvolvimento da agricultura nacional (EMBRAPA, 2005; COUTINHO, 2006).Nas interações com as raízes de plantas, os microrganismos podem ser endofíticos, simbióticos ou de vida livre. Entre aqueles de vida livre, destacam-se os microrganismos solubilizadores de fosfato (MSP), que são bactérias ou fungos capazes de solubilizar diferentes formas de fosfato de baixa solubilidade, deixando-o disponível para as plantas (BAREA et al., 2005). A habilidade dos microrganismos em solubilizar o $\mathrm{P}$ de várias fontes de $\mathrm{P}$ insolúveis, orgânicas e inorgânicas, tem sido considerada uma desejável característica para seu uso diverso (RICHARDSON et al., 2005).

Pereira et al. (2012) concluíram que existe correlação positiva entre a quantidade de fungos solubilizadores de fósforo presente em um solo, com a respectiva quantidade de $\mathrm{P}$ disponível. Em diferentes estudos os gêneros Aspergillus e Penicillium são os mais frequentes em solos de ambientes tropicais (CAVALCANTI et al., 2006; COUTINHO et al., 2010; FRAGA; PEREIRA, 2010; FRAGA et al., 2012; GRISHKAN; NEVO, 2008). Guimarães et al. (2006) afirmam serem estes gêneros os mais promissores no tocante à obtenção de fitase extracelular. A fitase pode ser intracelular ou extracelular, sendo a última a única potencialmente explorável do ponto de vista industrial. Os fungos filamentosos mostraram-se predominantes na produção da fitase extracelular, o que permite inferir que um estudo aprofundado, destes organismos em especial, poderia trazer resultados promissores. Além do fato de serem pouco explorados, há de se considerar que muitos destes seres possuem uma capacidade de sobrevivência e colonização em ambientes inóspitos, muito acima da maioria dos seres vivos (ABREU; PFENNING, 2008), o que constitui um forte indício de que, provavelmente, os mesmos possuem enzimas e outros componentes metabólicos altamente eficientes, ou que têm determinadas características peculiares potencialmente exploráveis em atividades agropecuárias e industriais. Estes fatores levam a crer que os fungos de solos, especialmente os filamentosos, que têm demonstrado particular potencial de exploração, devem ter seu estudo intensificado e ampliado. A partir do exposto, este trabalho teve como objetivo caracterizar a micobiota produtora de fitase no Bioma Cerrado, bem como, apresentar uma caracterização dos fatores abióticos do presente bioma e as possíveis correlações com a micobiota encontrada.

\section{Material e métodos}

O estudo foi realizado no Bioma Cerrado, situado entre as Latitudes: $19^{\circ} 26^{\prime} 10^{\prime \prime}$ e $19^{\circ} 27^{\prime} 38^{\prime \prime}$ e as Longitudes: $44^{\circ} 08^{\prime} 52^{\prime \prime}$ e $44^{\circ} 11^{\prime} 10^{\prime \prime}$ em Sete Lagoas-MG com altitude média de $710 \mathrm{~m}$, em que 
foram selecionados de forma aleatória oito fragmentos florestais, característicos do Bioma em estudo. Nestes fragmentos foram realizadas coletas de amostras de serapilheira e solo, por meio do método de parcelas (MUELLER-DOMBOIS; ELLENBERG, 1974), com parcelas de $20 \mathrm{~m} \times 20$ $\mathrm{m}$, orientadas no sentido N-S, L-O, distantes $100 \mathrm{~m}$ da borda do fragmento. A altura médias das árvores dos diferentes fragmentos se manteve entre um mínimo de 8,62 metros e um máximo de $12,07 \mathrm{~m}$, enquanto a declividade média do terreno (em graus) entre e $5^{\circ}$ e $26,5^{\circ}$. Já o Índice de Shannon esteve entre 2,19 e 2,92.

As amostras de serapilheira e solo foram coletadas em duas diferentes épocas, final de janeiro de 2012 (C1), e início de setembro de 2012 (C2). A primeira coleta foi realizada três dias após um período de quatro dias de intensa chuva, que totalizou 101,2 $\mathrm{mm}$ de precipitação pluviométrica. A segunda coleta foi realizada 22 dias após uma chuva atípica de $0,4 \mathrm{~mm}$, ocorrida durante o período de estiagem. Foram amostrados os oito fragmentos selecionados, sendo que um deles foi subdividido em dois sítios, em virtude do tamanho do mesmo. Foram retiradas três amostras compostas, formadas a partir de três amostras simples, camada de serapilheira e solo nas profundidades de 0-5 e 5-10 cm.

A temperatura média (Tmed) mensal do período chuvoso foi $22,8^{\circ} \mathrm{C}$, enquanto as médias mensais das temperaturas máximas (Tmax) e mínimas (Tmin) diárias foram $29^{\circ} \mathrm{C}$ e $18^{\circ} \mathrm{C}$, respectivamente. Já para o período de seca o Tmed foi $18,7^{\circ} \mathrm{C}$, enquanto Tmax e Tmin foram $26,8^{\circ} \mathrm{C}$ e $12^{\circ} \mathrm{C}$, respectivamente. A temperatura máxima registrada no mês de janeiro foi $35,4^{\circ} \mathrm{C}$ e a mínima foi $15,1^{\circ} \mathrm{C}$. Para o mês de setembro foram $31,8^{\circ} \mathrm{C}$ para Tmax e $8^{\circ} \mathrm{C}$ para $\mathrm{Tmin}$.

A precipitação pluvial média $(\mathrm{Ppm})$ ao longo de $\mathrm{C} 1$ foi $12,37 \mathrm{~mm}$, enquanto a evapotranspiração média (Evpm) foi $2,42 \mathrm{~mm}$, totalizando um balanço hídrico médio (Bhm) de $9,95 \mathrm{~mm}$, consequentemente, $298,5 \mathrm{~mm}$ de chuva se acumularam na região. A média diária da umidade relativa do ar no mês de janeiro variou entre $55 \%$ e $97 \%$, enquanto no mês de setembro entre $32 \%$ e $74 \%$. Para C2 houve um balanço hídrico negativo.

Os isolamentos da micobiota foram feitos a partir de amostras de $5 \mathrm{~g}$ de solo e serapilheira ressuspendidas em $45 \mathrm{~mL}$ de $0,1 \%$ de peptona contendo $0,1 \%$ de Tween $80 \mathrm{em}$ frasco Erlenmeyer, em seguida agitadas por 30 min em homogeneizador de haste vertical a $150 \mathrm{rpm}$. O meio de cultura utilizado foi Dicloran Rosa de Bengal Cloranfenicol (DRBC), realizado com alíquotas de $0,1 \mathrm{~mL}$ das respectivas diluições $\left(10^{2}\right.$ a $\left.10^{6}\right)$. As placas de Petri foram incubadas em estufas a $25^{\circ} \mathrm{C}$ por 5 dias. A micobiota foi quantificada por unidade formadora de colônia (UFC) (FRAGA; PEREIRA, 2010).

Os testes para solubilização de fósforo foram realizados em meio de cultura Pikovskaya, acondicionadas em estufa a $25^{\circ} \mathrm{C}$ por um período de até 10 dias. As colônias que cresceram formando halos em seus entornos no respectivo meio de cultura foram consideradas produtoras da enzima fitase (PIKOVSKAYA, 1948; GOMES, 2010).

A fertilidade do solo foi avaliada através dos seguintes atributos: $\mathrm{pH}$ em água (método do potenciômetro), teor de $\mathrm{Al}$ (trocável), $\mathrm{H}+\mathrm{Al}, \mathrm{P}, \mathrm{K}, \mathrm{Ca}$ e $\mathrm{Mg}$ (trocáveis) para profundidades de 0-5 e 5-10 cm para solo e a camada de serapilheira. Os métodos de análise de solos foram os adotados por Embrapa (1997) (Tabela 1).

A análise de componentes principais (ACP) a partir da matriz de correlações para os valores médios de atributos de solo: $\mathrm{pH}$ em $\mathrm{H}_{2} \mathrm{O}, \mathrm{P}, \mathrm{K}, \mathrm{Ca}, \mathrm{Mg}, \mathrm{Al}, \mathrm{H}+\mathrm{Al}$ e matéria orgânica em cada uma das áreas amostrais (de F1 a F8, sendo a última subdividida em F8.1 e F8.2). A análise de ACP foi realizada por meio do ambiente $\mathrm{R}$, cujas variáveis apresentaram correlação de Pearson maiores que 0,7 entre si, relacionadas aos atributos químicos do solo. A ACP é utilizada para reduzir as dimensões dos dados e, consequentemente, facilitar a análise por meio do gráfico. A ACP condensa informações de um grande número de variáveis em um grupo menor de diferentes componentes (eixos) dimensionais, distorcendo o mínimo possível seu arranjo original (FELFILI et al., 2011). 


\section{Tabela 1 - Dados químicos do solo dos fragmentos F1 a F8.}

Table 1 - Soil chemical data from fragments F1 to F8.

\begin{tabular}{ccccccccc}
\hline $\mathbf{F}$ & $\mathbf{p H} \mathbf{H}_{2} \mathbf{O}$ & $\mathbf{P}$ & $\mathbf{K}$ & $\mathbf{C a}$ & $\mathbf{M g}$ & $\mathbf{A L}$ & H_AL & Matéria Orgânica \\
\hline $\mathbf{1}$ & 6,2 & 11,6 & 166,3 & 8 & 1,6 & 0 & 5,4 & 10,1 \\
$\mathbf{2}$ & 5,2 & 3,9 & 91,7 & 1,6 & 0,4 & 0,8 & 9,1 & 5,5 \\
$\mathbf{3}$ & 5,6 & 8,6 & 44,3 & 5,9 & 0,4 & 0 & 6 & 6,5 \\
$\mathbf{4}$ & 5,9 & 13,3 & 255 & 6,6 & 1,7 & 0 & 6,9 & 12,4 \\
$\mathbf{5}$ & 6 & 4,1 & 133 & 6,3 & 1,8 & 0 & 5,4 & 9,5 \\
$\mathbf{6}$ & 5,8 & 9,7 & 182,3 & 8,1 & 1 & 0 & 9 & 16,5 \\
$\mathbf{7}$ & 5,7 & 10,7 & 203,5 & 8,2 & 1,3 & 0,1 & 5,5 & 7,6 \\
$\mathbf{8 . 1}$ & 5 & 8,4 & 149,7 & 2,8 & 1,1 & 0,4 & 8,2 & 7,8 \\
$\mathbf{8 . 2}$ & 7,1 & 11,3 & 452,7 & 10 & 1,6 & 0 & 0,8 & 8,5 \\
\hline
\end{tabular}

Em que: $\mathrm{P}, \mathrm{K}, \mathrm{Ca}, \mathrm{Mg}, \mathrm{Al}, \mathrm{H}+\mathrm{Al}$ e Mat. org. = mg/dm³ $; \mathrm{F}=$ Fragmento florestal.

A análise de componentes principais (ACP) a partir da matriz de correlações para os valores médios de atributos de solo: $\mathrm{pH}$ em $\mathrm{H}_{2} \mathrm{O}, \mathrm{P}, \mathrm{K}, \mathrm{Ca}, \mathrm{Mg}, \mathrm{Al}, \mathrm{H}+\mathrm{Al}$ e matéria orgânica em cada uma das áreas amostrais (de F1 a F8, sendo a última subdividida em F8.1 e F8.2). A análise de ACP foi realizada por meio do ambiente $\mathrm{R}$, cujas variáveis apresentaram correlação de Pearson maiores que 0,7 entre si, relacionadas aos atributos químicos do solo. A ACP é utilizada para reduzir as dimensões dos dados e, consequentemente, facilitar a análise por meio do gráfico. A ACP condensa informações de um grande número de variáveis em um grupo menor de diferentes componentes (eixos) dimensionais, distorcendo o mínimo possível seu arranjo original (FELFILI et al., 2011).

\section{Resultados e discussão}

A quantificação da micobiota na época chuvosa revelou valores médios situados entre um mínimo de 7,8 $\times 10^{5}$ UFC e máximo de $16 \times 10^{5}$ UFC para a serapilheira; $1,9 \times 10^{5}$ e $6,0 \times 10^{5}$ UFC para a profundidade de $0-5 \mathrm{~cm}$ do solo; e $2,0 \times 10^{5}$ e $7,3 \times 10^{5}$ UFC para a profundidade de $5-10$ $\mathrm{cm}$ do solo. A média das médias, da serapilheira e das duas profundidades anteriormente citadas foram, respectivamente, $12 \times 10^{5} ; 4$ e $3,1 \times 10^{5} \mathrm{UFC}$.

A serapilheira apresentou número de UFC superior às demais profundidades (Figura 1). Para a profundidade de $0-5 \mathrm{~cm}$ verificou-se um maior número de UFC em comparação com a profundidade de 5-10 cm. Esse aumento do número de UFC da serapilheira em relação ao solo também foi observado por Fraga et al. (2012) ao estudarem a interação entre microrganismo, solo e flora na Mata Atlântica, Paracambi-RJ e Osaki e Netto (2013) ao avaliarem a distribuição vertical e horizontal da população fúngica em Floresta Ombrófila Mista, Tijucas-PR. A serapilheira depositada sobre o solo apresenta quantidade significante de nutrientes, que são reciclados principalmente pela decomposição de fungos, ambiente com melhor desenvolvimento e propagação para os fungos (FRAGA et al., 2012). 
Figura 1 - Quantificação da micobiota na época chuvosa (C1) dos fragmentos florestais em UFC $\left(\mathbf{1 0}^{5}\right)$ nas três profundidades avaliadas.

Figure 1 - Quantification of mycoflora in the rainy season (C1) of forest fragments in UFC $\left(10^{5}\right)$ in the three studied depths.

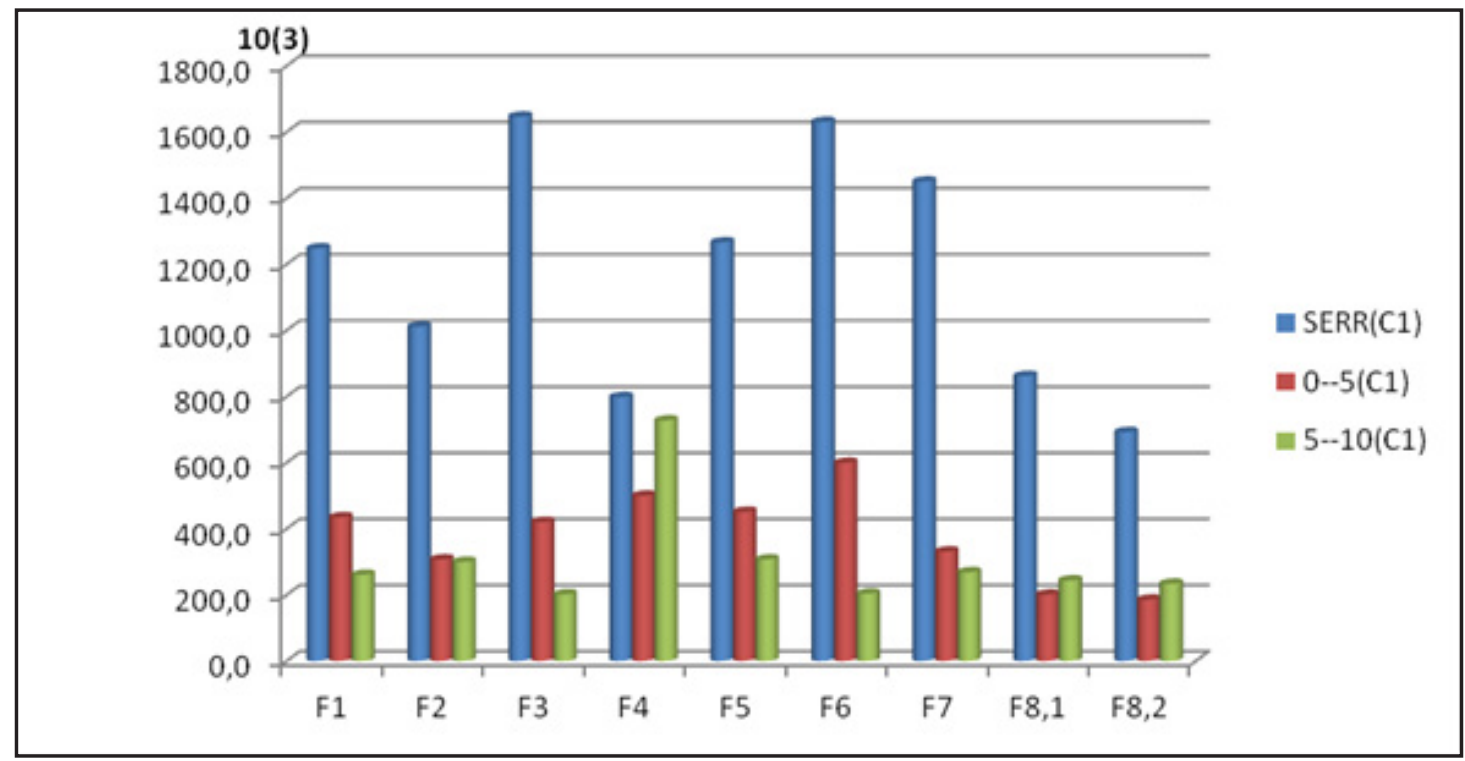

Fonte: Autores (2019)

Legenda: SERR = Serapilheira, 0-5 = profundidade de 0-5 $\mathrm{cm}$ do solo, 5-10 = profundidade de 5-10 $\mathrm{cm}$ do solo. Legend: SERR = burlap, $0-5=$ depth of $0-5 \mathrm{~cm}$ soil, $5-10=$ depth of $5-10 \mathrm{~cm}$ soil.

A quantificação da micobiota na época seca revelou valores médios situados entre um mínimo de 3,7 × $10^{5}$ UFC e máximo de $6,7 \times 10^{5}$ UFC para a serapilheira; $2,2 \times 10^{5}$ e 4,6 $\times 10^{5}$ UFC para a profundidade de $0-5 \mathrm{~cm}$ do solo; e $2 \times 10^{5}$ e $2,8 \times 10^{5}$ UFC para a profundidade de $5-10 \mathrm{~cm}$. A média das médias das UFC da serapilheira e das duas profundidades anteriormente citadas foram, respectivamente, $6,6 \times 10^{5} ; 3,0 \times 10^{5}$ e $2,3 \times 10^{5}$.

A serapilheira apresentou número de UFC superior às demais profundidades (Figura 2), e na profundidade de $0-5 \mathrm{~cm}$ observou-se um número de UFC pouco superior à profundidade de 5-10 cm. Em contrapartida, Osaki e Netto (2013), quando compararam os números de UFC das profundidades denominadas por eles de "zona de transição" (serapilheira) e solo, não encontraram diferenças entre o número de UFC destas duas camadas.

Quando comparadas as mesmas profundidades em diferentes épocas de coleta, as profundidades de serapilheira apresentaram maiores valores de UFC na época chuvosa. À diferença de UFC entre a serapilheira na estação úmida em comparação à seca foi superior a $81 \%$, enquanto para as diferentes profundidades do solo essa variação não ultrapassou $25 \%$ de diferença da época chuvosa à época seca, sendo os maiores valores verificados na época úmida (Figuras 1 e 2). Diferindo do padrão observado nesse estudo, Fraga et al. (2012) e Osaki e Netto (2013) não verificaram diferença entre o número de UFC das amostras coletadas no verão e inverno, o que corrobora a afirmativa que o principal fator para a diferença entre número de UFC das populações fúngicas coletadas nas diferentes estações neste estudo, seja, de fato, a diferença de umidade no solo entre as duas épocas, pois nos biomas estudados por estes autores citados, não foi observada uma diferença entre a pluviosidade das épocas de verão e inverno tão grande quanto as verificadas neste estudo.

Santos e Camargo (1999) afirmam que não só a temperatura como a umidade do solo influencia nas relações fisiológicas e nos atributos físicos e químicos do solo, e consequentemente 
no ambiente em que vivem os microrganismos. Souto (2006) demonstra que a população fúngica apresenta decréscimos consideráveis em função da diminuição do conteúdo de água no solo.

\section{Figura 2 - Quantificação da micobiota na época seca (C2) dos fragmentos florestais em UFC $\left(\mathbf{1 0}^{5}\right)$ nas três profundidades avaliadas.}

Figure 2 - Quantification of mycobiota in the dry season (C2) of forest fragments in UFC $\left(10^{5}\right)$ in the three studied depths.

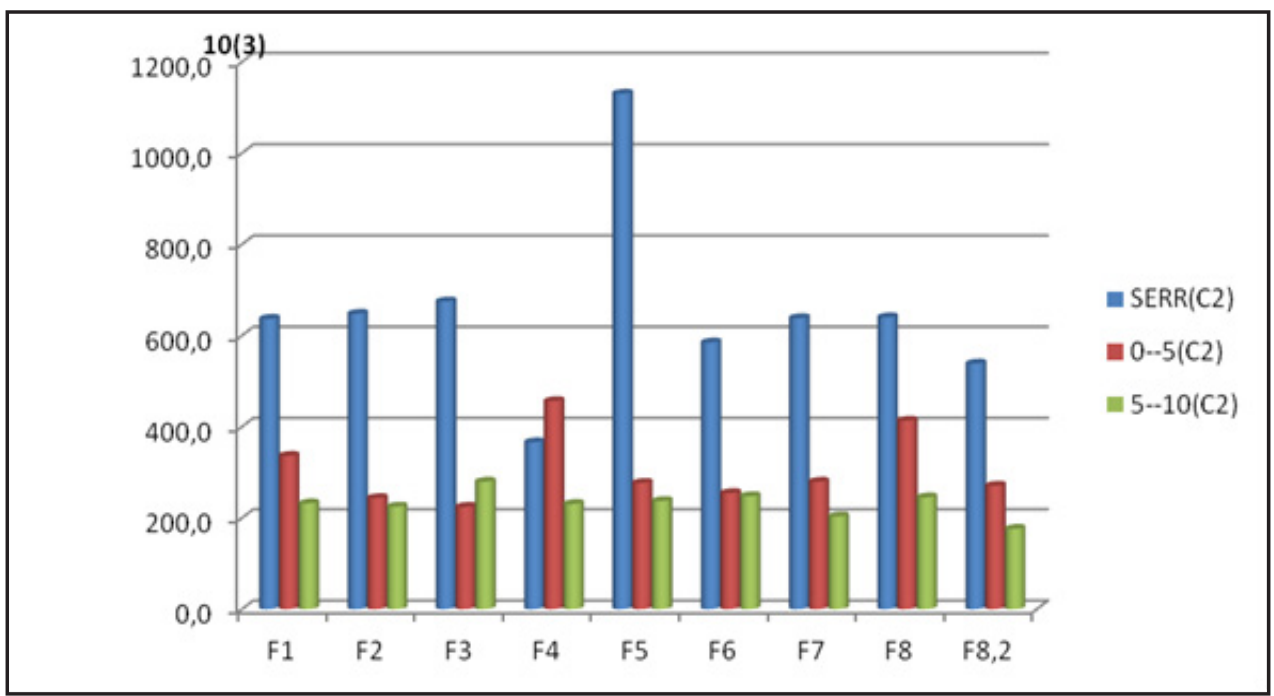

Fonte: Autores (2019)

Legenda: $S E R R=$ Serapilheira, $0-5=$ profundidade de $0-5 \mathrm{~cm}$ do solo, 5-10 = profundidade de 5-10 $\mathrm{cm}$ do solo. Legend: SERR = burlap, 0-5 = depth of 0-5 cm soil, 5-10 = depth of 5-10 cm soil.

Os padrões observados neste estudo corroboram os trabalhos de Wellbaum (2006) e Fraga e Pereira (2010), em estudo da micobiota do solo de Pinus verificou-se um aumento progressivo das UFCs em função do aumento da precipitação pluviométrica. Contudo, os resultados de Silva Reis et al. (2013) sugerem que a interferência da quantidade de água presente no solo na composição quantitativa dos fungos fica restrita até um determinado limite, a partir do qual seu aumento passa a não ter efeitos na micobiota, uma vez que tais resultados concluíram não haver diferença entre a quantidade de fungos presentes no solo obtida nas diferentes lâminas irrigadas.

$\mathrm{Na}$ estação chuvosa apenas $2,3 \%$ dos fungos solubilizadores de $\mathrm{P}$, produtores de fitase não pertenciam ao gênero Aspergillus e Penicillium. Enquanto em estação seca apenas 2,8\%. Os demais gêneros produtores de fitase encontrados foram: Trichoderma, Paecilomyces, Acremonium, Fusarium, Cladosporium. Somente em C2 foram encontrados Acremonium, Fusarium e Cladosporium. A distribuição destes últimos, assim como dos Aspergillus spp não produtores de fitase, é apresentada nas Tabelas 2 e 3.

Na coleta C1, foram isolados 907 fungos, destes, 103 (11,35\%) apresentaram capacidade de solubilizar P; 121 (13,34\%) pertenciam ao gênero Trichoderma; $10(1,1 \%)$ pertenciam ao gênero Aspergillus sem solubilizar P (AspNPF); 48 pertenciam ao gênero Penicillium e apresentavam capacidade de solubilizar $\mathrm{P}$ e 40 pertenciam à seção Nigri do gênero Aspergillus e apresentavam capacidade de solubilizar P (AspFnig) (Tabela 2).

Os números de fungos pertencentes ao gênero Penicillium produtores de fitase e o número de isolados pertencentes ao gênero Trichoderma foram semelhantes, porém, estes foram superiores ao número de Aspergillus não produtores de fitase e Aspergillus produtores de fitase pertencentes à seção Nigri. Fraga et al. (2012) e Santos et al. (2007) também encontraram distribuições numéricas distintas entre os grupos estudados. 
Na coleta C2, foram isolados 947 fungos, $152(16,76 \%)$ apresentando a capacidade de solubilizar P, 131 (13,83\%) de Trichoderma, 17 (1,8\%) de Aspergillus sem solubilizar P (AspNPF), 93 de Penicillium e apenas 3 de Aspergillus seção Nigri (AspFnig) (Tabela 3).

Tabela 2 - Distribuição numérica dos principais grupos fúngicos avaliados ao longo dos fragmentos florestais na época chuvosa.

Table 2 - Numerical distribution of the main fungal groups evaluated along the forest fragments in the rainy season.

\begin{tabular}{|c|c|c|c|c|c|c|}
\hline Fragmento & Profundidade & Penicillium & Trichoderma & Fitase & AspNPF & AspFnig \\
\hline \multirow{3}{*}{1} & S & 2 & 5 & 4 & 1 & 0 \\
\hline & $0-5 \mathrm{~cm}$ & 3 & 3 & 5 & 0 & 2 \\
\hline & $5-10 \mathrm{~cm}$ & 2 & 5 & 3 & 0 & 3 \\
\hline \multirow{3}{*}{2} & S & 0 & 0 & 1 & 0 & 1 \\
\hline & $0-5 \mathrm{~cm}$ & 2 & 3 & 4 & 1 & 2 \\
\hline & $5-10 \mathrm{~cm}$ & 1 & 2 & 3 & 0 & 2 \\
\hline \multirow{3}{*}{3} & S & 1 & 1 & 0 & 0 & 0 \\
\hline & $0-5 \mathrm{~cm}$ & 2 & 3 & 2 & 0 & 0 \\
\hline & $5-10 \mathrm{~cm}$ & 1 & 2 & 2 & 0 & 0 \\
\hline \multirow{3}{*}{4} & $S$ & 1 & 0 & 1 & 0 & 1 \\
\hline & $0-5 \mathrm{~cm}$ & 1 & 2 & 3 & 0 & 1 \\
\hline & $5-10 \mathrm{~cm}$ & 0 & 3 & 2 & 0 & 1 \\
\hline \multirow{3}{*}{5} & S & 2 & 1 & 3 & 1 & 2 \\
\hline & $0-5 \mathrm{~cm}$ & 5 & 3 & 6 & 0 & 2 \\
\hline & $5-10 \mathrm{~cm}$ & 4 & 3 & 7 & 0 & 0 \\
\hline \multirow{3}{*}{6} & S & 2 & 1 & 2 & 0 & 0 \\
\hline & $0-5 \mathrm{~cm}$ & 2 & 2 & 4 & 0 & 1 \\
\hline & $5-10 \mathrm{~cm}$ & 2 & 0 & 4 & 0 & 1 \\
\hline \multirow{3}{*}{7} & $S$ & 1 & 2 & 0 & 0 & 0 \\
\hline & $0-5 \mathrm{~cm}$ & 0 & 3 & 4 & 0 & 2 \\
\hline & $5-10 \mathrm{~cm}$ & 0 & 0 & 1 & 0 & 0 \\
\hline \multirow{3}{*}{8} & $S$ & 1 & 7 & 5 & 0 & 4 \\
\hline & $0-5 \mathrm{~cm}$ & 1 & 18 & 7 & 1 & 7 \\
\hline & $5-10 \mathrm{~cm}$ & 1 & 10 & 11 & 0 & 8 \\
\hline
\end{tabular}

Em que: AspNPF = Aspergillus não produtores de fitase, AspFnig = Aspergillus produtores de fitase da seção Nigri.

Nas amostras da estação seca $19,5 \%$ dos fungos solubilizadores de $\mathrm{P}$ foram isolados da serapilheira, enquanto as profundidades de 0-5 e 5-10 cm responderam por 46,6 e 33,9\%, respectivamente. Na estação chuvosa, a serapilheira contribuiu com $19 \%$ do total de fungos produtores de fitase, e as demais profundidades forneceram $80,95 \%$, havendo pouca diferença 
no percentual destas. Foi observado que, do total de fungos solubilizadores de $\mathrm{P}$ que pertenciam à seção Nigri do gênero Aspergillus, 20,9\% foram provenientes da camada de serapilheira, $41,9 \%$ da profundidade de $0-5 \mathrm{~cm}$, e $37,20 \%$ de $5-10 \mathrm{~cm}$ do solo. Diferindo do padrão encontrado neste estudo, Ramos et al. (2012) concluíram que na camada de $5-20 \mathrm{~cm}$ do solo o número de microrganismos solubilizadores de $\mathrm{P}$ é superior tanto na estação seca como na chuvosa em Bioma Cerrado.

Tabela 3 - Distribuição numérica dos diferentes grupos fúngicos avaliados ao longo dos fragmentos florestais na época seca.

Table 3 - Numerical distribution of different fungal groups evaluated along the forest fragments in the dry season.

\begin{tabular}{|c|c|c|c|c|c|c|}
\hline Fragmento & Profundidade & Penicillium & Trichoderma & Fitase & AspNPF & AspFnig \\
\hline \multirow{3}{*}{1} & $S$ & 2 & 4 & 6 & 1 & 0 \\
\hline & $0-5 \mathrm{~cm}$ & 10 & 8 & 5 & 1 & 0 \\
\hline & $5-10 \mathrm{~cm}$ & 6 & 6 & 11 & 0 & 0 \\
\hline \multirow{3}{*}{2} & S & 2 & 2 & 1 & 0 & 0 \\
\hline & $0-5 \mathrm{~cm}$ & 4 & 1 & 4 & 1 & 1 \\
\hline & $5-10 \mathrm{~cm}$ & 3 & 2 & 4 & 1 & 0 \\
\hline \multirow{3}{*}{3} & S & 2 & 5 & 2 & 1 & 0 \\
\hline & $0-5 \mathrm{~cm}$ & 3 & 2 & 5 & 0 & 0 \\
\hline & $5-10 \mathrm{~cm}$ & 2 & 2 & 2 & 0 & 0 \\
\hline \multirow{3}{*}{4} & $S$ & 2 & 2 & 2 & 0 & 0 \\
\hline & $0-5 \mathrm{~cm}$ & 7 & 4 & 8 & 0 & 0 \\
\hline & $5-10 \mathrm{~cm}$ & 3 & 3 & 5 & 0 & 1 \\
\hline \multirow{3}{*}{5} & S & 4 & 8 & 3 & 1 & 0 \\
\hline & $0-5 \mathrm{~cm}$ & 5 & 12 & 13 & 0 & 0 \\
\hline & $5-10 \mathrm{~cm}$ & 5 & 10 & 5 & 0 & 0 \\
\hline \multirow{3}{*}{6} & S & 1 & 3 & 2 & 0 & 0 \\
\hline & $0-5 \mathrm{~cm}$ & 4 & 5 & 5 & 0 & 0 \\
\hline & $5-10 \mathrm{~cm}$ & 4 & 3 & 5 & 0 & 0 \\
\hline \multirow{3}{*}{7} & $S$ & 1 & 2 & 3 & 0 & 1 \\
\hline & $0-5 \mathrm{~cm}$ & 3 & 5 & 6 & 0 & 0 \\
\hline & $5-10 \mathrm{~cm}$ & 4 & 3 & 2 & 0 & 0 \\
\hline \multirow{3}{*}{8} & $S$ & 8 & 2 & 4 & 0 & 0 \\
\hline & $0-5 \mathrm{~cm}$ & 5 & 7 & 9 & 1 & 0 \\
\hline & $5-10 \mathrm{~cm}$ & 3 & 7 & 11 & 1 & 0 \\
\hline
\end{tabular}

Em que: AspNPF = Aspergillus não produtores de fitase; AspFnig = Aspergillus produtores de fitase da seção Nigri.

O número de isolados produtores de fitase pertencentes ao gênero Penicillium na estação 
seca foi maior que na estação chuvosa. Isso pode ser explicado pela biossíntese das enzimas mineralizadoras de fitato, na maioria dos microrganismos, é induzida por carência de $\mathrm{P}$ no solo, e nesta estação, devido à maior restrição hídrica, este elemento está menos disponível. Segundo Ireland et al. (2002), é um indicativo o papel da fitase em prover P para as células. O segundo motivo seria que nos solos, existe uma maior riqueza de espécies de Penicillium no período seca do ano, indicando que as espécies deste gênero são bem adaptadas a solos com baixa disponibilidade de água (SILVA, 2013).

$\mathrm{Na}$ estação chuvosa, dos fungos isolados $(19,4 \%)$ pertenciam à seção Nigri do gênero Aspergillus, enquanto na estação seca este percentual foi de apenas 1,4\%. Esse padrão corrobora os dados de Pitt e Hocking (1997), os quais afirmam que os Aspergillus negros se desenvolvem melhor em temperaturas elevadas, um pouco acima da temperatura ambiente.

O número de fungos do gênero Trichoderma isolados na época seca foi superior aos isolados na época chuvosa, o que está de acordo com Santos et al. (2007), que afirmam que o excesso de umidade no solo não favorece o desenvolvimento dos fungos deste gênero, e que os aspectos climáticos provocam maiores variações nas populações fúngicas.

$\mathrm{Na}$ análise de componentes principais (ACP), os dois primeiros componentes da análise foram capazes de explicar $77,92 \%$ da variância acumulada das variáveis em questão para as áreas amostradas. Pode-se afirmar que existe correlação negativa do $\mathrm{pH}$ e $\mathrm{K}$ do solo com a acidez potencial $(\mathrm{H}+\mathrm{Al})$, assim como entre $\mathrm{P}$ e $\mathrm{Al}$. Os íons $\mathrm{Ca}$ e $\mathrm{Mg}$ distribuem-se da mesma forma nas áreas, assim como os valores de $\mathrm{pH}$ e os teores de K. Não foi observada a formação de nenhum grupo de fragmentos florestais similares dentro das dimensões avaliadas (Figura 3).

Não foi possível verificar qualquer relação entre os atributos da fertilidade do solo dos fragmentos florestais com seus respectivos números médios de UFC. Os cinco fragmentos com maiores teores de nutrientes e menores de elementos tóxicos ( $\mathrm{Al}$ ) foram, em ordem decrescente: F8, F1, F4, F5 e F7, enquanto os cinco fragmentos com os maiores números de UFC foram: F5, F6, F3, F7 e F1. Correlações entre a quantidade de fósforo presente nos solos e seu respectivo número de UFC seriam esperadas, pois, de acordo com Sylvia et al. (1999), N e P são os principais nutrientes exigidos no metabolismo dos organismos devido às demandas estruturais e funcionais das células. Ferreira et al. (2008) concluíram que a adição de $\mathrm{P}$ ao solo afeta o metabolismo de sua microbiota, fato este indicado pelo aumento expressivo da liberação de $\mathrm{CO}_{2}$. Contudo, os fragmentos que apresentaram os maiores teores de P foram: F4, F1, F8, F7 e F6, enquanto os que apresentaram maiores números de UFC foram F5, F6, F3, F7 e F1, não sendo possível, portanto, afirmar que foi observada uma nítida correlação positiva entre teores de $\mathrm{P}$ e quantidade de UFC do solo.

Nenhum dos atributos químicos do solo apresentou correlação com qualquer grupo fúngico em qualquer profundidade avaliada. Esta ausência de correlação entre fatores atributos químicos do solo com quantidade de fungos solubilizadores de $\mathrm{P}$ também foi observada por Neves et al. (2010). Contudo, foi verificado que dos fragmentos florestais com os maiores valores de $\mathrm{pH}$ e matéria orgânica do solo, foram isoladas as maiores quantidades de fungos produtores de fitase, sendo os fragmentos com os maiores números de fungos produtores de fitase: F5, F1, F8 e F6, enquanto os fragmentos que apresentaram os maiores valores de $\mathrm{pH}$ foram F8, F1, F5 e F4, e os que apresentaram os maiores valores de matéria orgânica foram: F6, F4, F1 e F5. Entretanto, Nahas (2002) em um estudo quantificando a comunidade de microrganismos mineralizadores de $\mathrm{P}$ orgânico, produtores de fosfatases ácida e alcalina, bem como o efeito do tipo de espécie cultivada e o tipo de calagem efetuada sobre tal comunidade, concluiu que a realização da calagem diminui o número de fungos produtores de fosfatase, paralelo ao aumento do número de bactérias, o que pode ser considerado um indício de que, não apenas a fitase fúngica, como as demais enzimas fúngicas que atuam sobre o $\mathrm{P}$, não têm sua produção estimulada por ambientas ricos em P. Em contrapartida, tal estudo não encontrou relação entre adubação e a quantidade destes microrganismos estudados, o que corrobora o presente estudo.

Pereira et al. (2012), em estudo que objetivou testar o impacto das mudanças no uso dos 
solos do Bioma Pampa Brasileiro, sob a diversidade microbiana, concluíram que a quantidade de fosfato presente no solo estava negativamente correlacionada com a quantidade de microrganismos com capacidade de solubilização de P.

Os solos situados no Bioma Cerrado são potencialmente exploráveis no tocante à obtenção de fungos solubilizadores de fósforo, e que a época seca do ano é mais propícia à obtenção de fungos solubilizadores de fósforo.

Figura 3 - Análise de componentes principais dos atributos químicos nas diferentes áreas de estudo.

Figure 3 - Principal component analysis of chemicals in the different areas of study attributes.

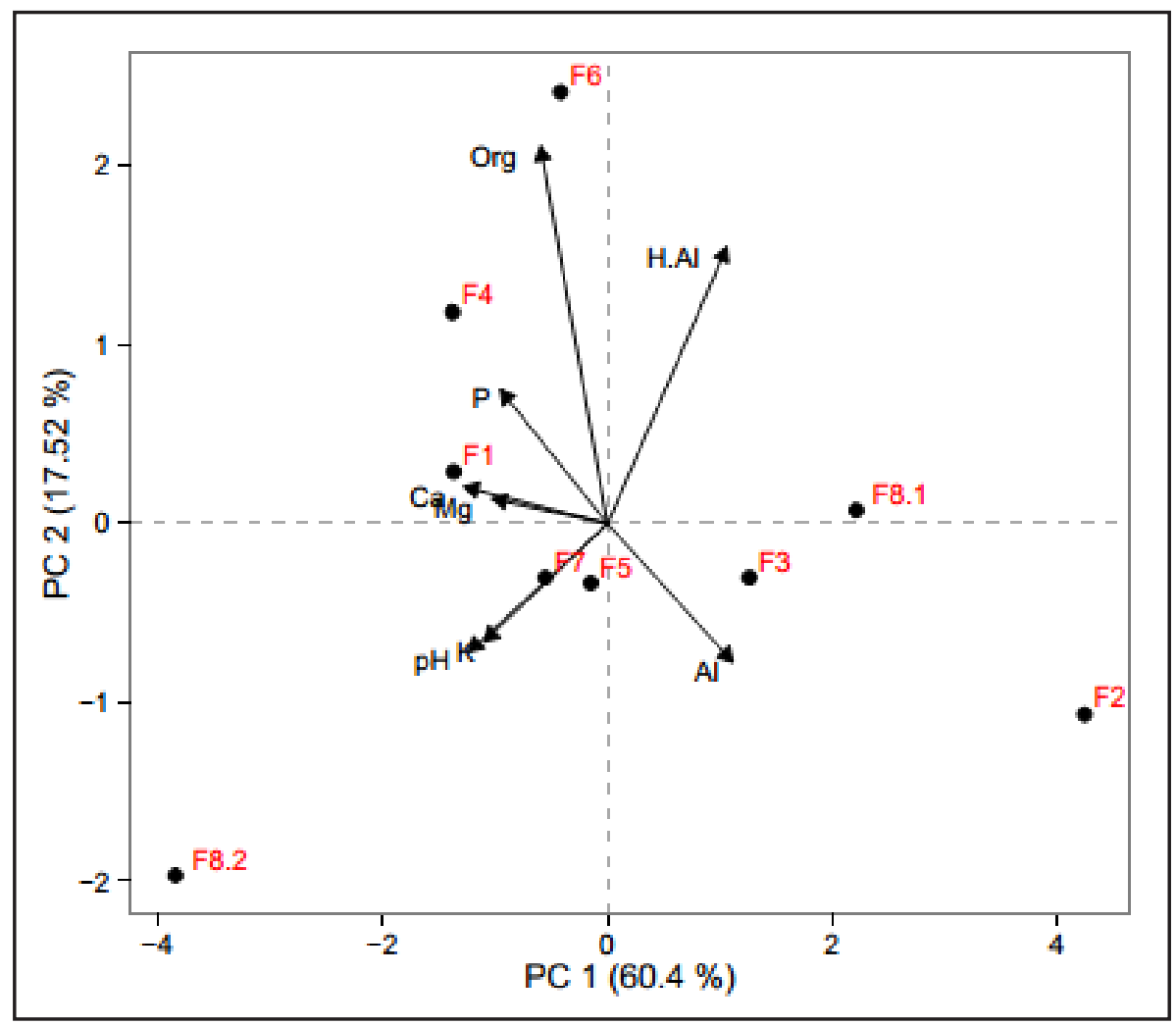

Fonte: Autores (2019)

\section{Conclusões}

O número de UFC da serapilheira foi superior ao das camadas de solo 0-5 e 5-10 cm, em ambas as estações de coleta.

A época de coleta interfere quantitativa e qualitativamente na composição da micobiota de um solo de Cerrado. O número total de fungos produtores de fitase foi maior na estação seca, assim como o número de fungos produtores de fitase pertencentes ao gênero Penicillium, contudo, o número de fungos pertencentes à seção Nigri do gênero Aspergillus produtores de fitase foi maior na época chuvosa.

Verificou-se alta variabilidade na fertilidade do solo entre as áreas. F5 e F7 podem ser consideradas áreas de condição intermediária de fertilidade, dada sua proximidade à origem do biplot. F2 destaca-se pelo relativo acúmulo de Al. F1, F4 e F6 encontram-se numa região de maior fertilidade, dada sua relação positiva com variáveis como $\mathrm{pH}, \mathrm{Ca}, \mathrm{Mg}, \mathrm{P}$ e matéria orgânica. 
F3 e F8.1 encontram-se em região sob maior influência do Al e H+Al. O F8.2 destaca-se por seus elevados valores de $\mathrm{pH}$ e $\mathrm{K}$, sendo a área de maior especificidade nutricional em termos de fertilidade do solo.

\section{Referências}

ABREU, L. M.; PFENNING, L. H. Diversidade de microfungos em solos tropicais. In: MOREIRA, F. M. S.; SIQUEIRA, J. O.; BRUSSAARD, L. (ed.). Biodiversidade do solo em ecossistemas brasileiros. Lavras: Ed. UFLA, 2008. p. 445-481.

BAREA, J. M. et al. Microbial cooperation in the rhizosphere. Journal Experimental Botany, Ghent, v. 56, p. 1761-1778, apr. 2005.

CAVAlCANTI, M. A. Q.; OliVEIRA, L. G.; FERNANDES, M. A.; LiMA, D. M. Fungos filamentosos isolados do solo em municípios da região Xingó, Brasil. Acta Botânica Brasílica, v. 20 , n. 4 , p. $831-837$, out./dez.2006.

COUTINHO, F. P. et al. Filamentous fungi isolated from rhizosphers of melon plants (Cucumis melo cv. Geld) cultivated in soil with organic amendments. Acta Botanica Brasilica, São Paulo, v. 24, p. 292-298, jan./mar. 2010.

COUTINHO, L. M. O conceito de bioma. Acta Botânica Brasílica, São Paulo, v. 1, n. 20, p. 13-23, jan./mar. 2006.

EMBRAPA. Embrapa Cerrados: conhecimento, tecnologia e compromisso ambiental. 2. ed. Planaltina, 2005. 43 p.

EMBRAPA. Manual de métodos de análise de solo. Rio de Janeiro: EMBRAPA Solos, 1997. 212 p.

FELFILI, J. M. et al. Fitossociologia no Brasil: métodos e estudos de casos. Viçosa, MG: Editora UFV, 2011. p. 122-155.

FERREIRA, A. et al. Atividade respiratória da microbiota e conteúdo de glicose em resposta à adição de fósforo em solo de cerrado. Revista Brasileira de Ciência do Solo, Viçosa, MG, v. 32, p. 1891-1897, set./out. 2008.

FRAGA, M. E. et al. Interação micro-organismos, solo e flora como condutores da diversidade na Mata Atlântica. Acta Botanica Brasilica, São Paulo, v. 26, p. 857-886, jan./fev. 2012.

FRAGA, M. E.; PEREIRA, M. G. Micobiota de solo de uma área de Duna na Restinga da Marambaia. Floresta e Ambiente, Seropédica, v. 17, p. 30-36, out./dez. 2010.

GOMES, E. A. et al. Prospecção de comunidades microbianas do solo ativas no aproveitamento agrícola de fontes de fósforo de baixa solubilidade. [S. l.]: EMBRAPA Milho e Sorgo, 2010. (Documentos, v. 107).

GRISHKAN, I.; NEVO, E. Soil microfungal communities of "Evolution Canyons" in Israel extreme differences on a regional scale. Biological Journal Linnean Society, London, v. 93, p. 157-163, jan. 2008.

GUIMARÃES, L. H. S. et al. Screening of filamentous fungi for production of enzymes of biotechnological interest. Brazilian Journal Microbiology, São Paulo, v. 37, p. 474-480, jan./mar. 2006.

GUSEWELL, S. N. P. Rations in terrestrial plants: variation and functional significance. New Phytologisty, Beijing, v. 164, p. 243-266, jun. 2004.

INTERNATIONAL FERTILIZES INDUSTRY ASSOCIATION. Fertilizers. 2006. Disponível em: <http://www.fertilizes.org>. Acesso em: 10 abr. 2014. 
IRELAND, M. M. et al. Proteomic analysis of the Caulobacter crescentus stalk indicates competence for nutrient uptake. Molecular Microbiology, Salem, v. 45, p. 1029-1041, aug. 2002.

MUELLER-DOMBOIS, E.; ELLENBERG, F. Aims and methods of vegetation ecology. New York: Wiley \& Sons, 1974. 168 p.

NAHAS, E. Microrganismos do solo produtores de fosfatases em diferentes sistemas agrícolas. Bragantia, Campinas, v. 61, p. 267-275, jul./set. 2002.

NEVES, A. A. O.; MARRIEL, I. E.; SCHAFFERT, R. E. Diversidade funcional e atividade de micro-organismos solubilizadores de $\mathrm{P}$ na rizosfera de linhagens recombinantes de sorgo. In: CONGRESSO NACIONAL DE MILHO E SORGO, Goiânia, 2010. Anais [...]. Goiânia: [s. l.], 2010. CD-ROM.

OSAKI, F.; NETTO, P. S. Flutuação da população de fungos sob floresta ombrófila mista e em povoamento de Pinus taeda. Floresta, Curitiba, v. 42, p. 795-808, out./ dez. 2013.

PEREIRA, T. P. et al. Reestruturação e perda de diversidade bacteriana no bioma Pampa brasileiro. In: SALÃO INTERNACIONAL DE ENSINO, PESQUISA E EXTENSÃO, Bagé, 2012. Anais [...]. Bagé: [s. l.], 2012. p. 15-18.

PIKOVSKAYA, R. I. Mobilization of phosphorus in soil in connection with vital activity of some microbial species. Microbiology, New York, v. 17, p. 362-370, 1948.

PITT, J. I. E.; HOCKING, A. D. Fungi and food spoilage. London: Blackie Academic \& Professional, 1997. 593 p.

RAMOS, M. L. G. et al. Effect of management systems and planting on functional microorganisms density, at Cerrado soil. Bioscience Journal, Uberlândia, v. 28, p. 58-68, jan./fev. 2012.

RICHARDSON, A. E. et al. Utilization of soil organic phosphorus by higher plants. In: TURNER, B. L.; FROSSARD, E.; BALDWIN, D. S. (ed.). Organic phosphorus in the environment. Wallingford: CABI Publishing, 2005. p. 165-184.

SANTOS, G. A.; CAMARGO, F. A. O. Fundamentos da matéria orgânica do solo: ecossistemas tropicais e subtropicais. Porto Alegre: Genesis, 1999. 245 p.

SANTOS, R. P. et al. Enriquecimento da coleção de agentes de controle biológico de fitopatógenos: novos isolados de Trichoderma procedentes de Petrolina (PE). Brasília: EMBRAPA Recursos Genéticos e Biotecnologia, 2007. 750 p.

SILVA R. J. B. R. et al. Efeito de lâminas de irrigação e doses de PBZ na microfauna do solo cultivado com mangueira cv. Haden no norte de Minas Gerais. Revista Brasileira de Agricultura e Irrigação, Fortaleza, v. 5, p. 1-12, set./out. 2013.

SOARES, M. R.; ALLEONI, L. R. F. Contribution of soil organic carbon to the ion exchange capacity of tropical soils. Journal of Sustainable Agriculture, Binghamton, v. 32, p. 439-462, out. 2008.

SOUTO, P. C. Acumulação e decomposição da serapilheira e distribuição de organismos edáficos em área de caatinga na Paraíba, Brasil. 2006. 110 f. Tese (Doutorado em Agronomia) Universidade Federal da Paraíba, João Pessoa, 2006.

SYLVIA, D. M. et al. Principles and applications of soil microbiology. New Jersey: Prentice Hall, 1999. 550 p.

WELlBAUM, C. Produção de ácidos graxos por linhagens de Mucor sp isoladas de solo de Cerrado, Município de Corumbataí, Estado de São Paulo. 2006. 204 f. Tese (Doutorado em Ciências Biológica) - Universidade Estadual Paulista Julio de Mesquita Filho, São Paulo, 2006. 